\title{
First trimester sonographic diagnosis of limb-body wall defect associating both cephalic and thoraco-abdominal defects - a case report and literature update
}

\author{
Dan Boitor-Borza, Adelina Staicu, Constantin Roxana, Daniel Mureşan
}

$1^{\text {st }}$ Department of Obstetrics and Gynecology, "Iuliu Haţieganu” University of Medicine and Pharmacy, Cluj-Napoca, Romania

\begin{abstract}
We present the first trimester prenatal ultrasonography and pathological assessment of a case diagnosed with limb-body wall complex (LBWC) presenting both exenchephaly and a complex thoraco-abdominal wall defect. Ectopia cordis is demonstrated with a movie showing the heart beating outside the body of the fetus after its expulsion. Also, we discuss the pathogenesis and possible etiology of LBWC and associated malformations and we provide an update of the literature of this very rare anomaly.
\end{abstract}

Keywords: limb-body wall complex; exencephaly; abdominal wall defects; thoracoschisis; ectopia cordis

\section{Introduction}

Limb-body wall complex (LBWC) is a rare, sporadic pattern of multiple malformations with reported prevalence of 0.03-3.3 per 10,000 live births [1]. The original diagnostic criteria were established by van Allen in 1987 as an association of two out of three following characteristics: limb defects of any type, thoracoschisis or/and abdominoschisis and exencephaly or encephalocoele with/ without facial clefts [2].

As a result of clinical observations, in 1993 Russo et al identified two distinct phenotypes: type I placentocranial and type II placento-abdominal [3]. Because this classification did not succeed to include all observations from clinical practice, in 2007 Sahinoglu et al proposed a new classification, describing three phenotypes [4].

Received 02.02.2021 Accepted 12.03.2021

Med Ultrason

2022, Vol. 24, No 2, 245-247

Corresponding author: Adelina Staicu, MD

$1^{\text {st }}$ Department of Obstetrics and Gynecology,

"Iuliu Haţieganu" University of

Medicine and Pharmacy, 3-5 Clinicilor Street,

400006 Cluj-Napoca, Romania

Phone/Fax: +40264-450 115

E-mail: adelina.staicu@umfcluj.ro
We present the first trimester prenatal ultrasound assessment and pathological confirmation of a rare case of LBWC, which presented combined phenotypic features, as it was associated with both exenchephaly and a complex thoraco-abdominal wall defect. Our case respected Van Allen criteria but was distinct from the fetuses observed by Russo et al and Sahinoglu et al $[3,4]$.

To the best of our knowledge, this is the first case in literature exposing combine cephalic and thoraco-abdominal characteristics describe by ultrasound in the first trimester.

\section{Case report}

A 31-year-old healthy, Caucasian woman, gravida 2 para 1 , presented for routine prenatal sonographic examination at 10 weeks and 5 days of amenorrhea. The patient's personal history revealed a healthy child from a previous normal pregnancy. The current pregnancy was spontaneous and no preconception exposures to teratogenic agents, recent infections or drug intake reported. Family medical history of congenital malformations and blood tests were not remarkable.

Transvaginal sonography was performed on a GE Voluson E6 machine (GE Medical systems, Kretztechnik 


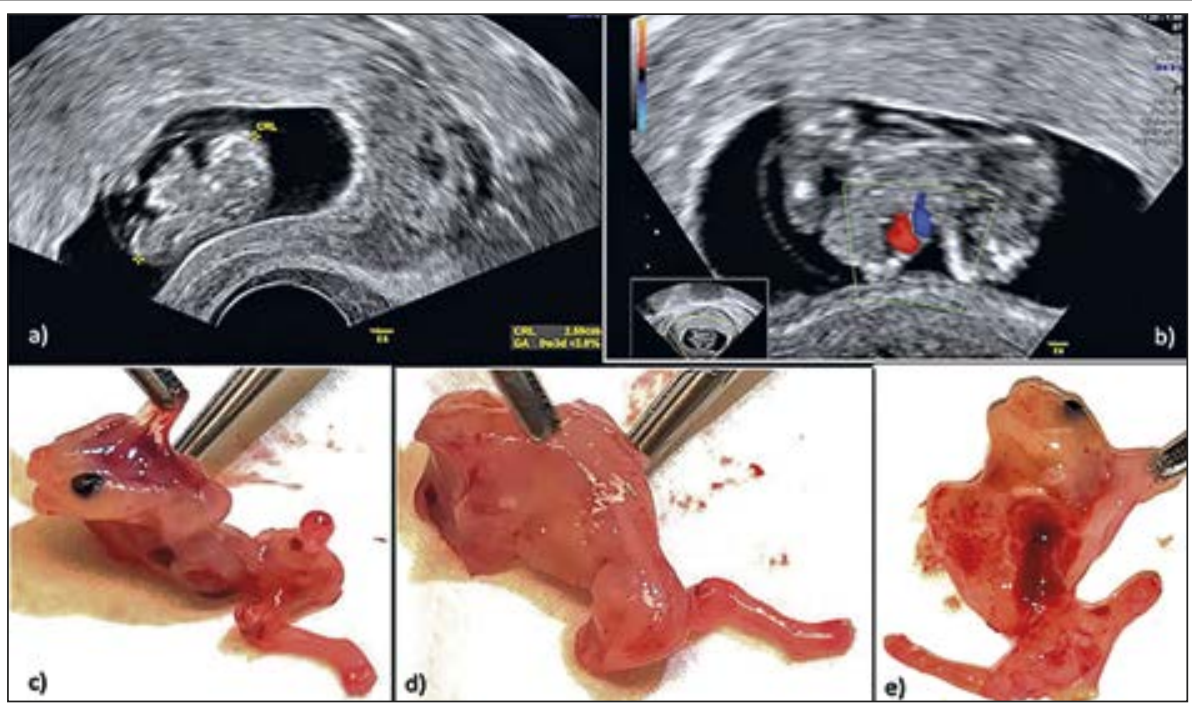

Fig 1. Ultrasound examination depicting a first trimester monoembrionar pregnancy with a plurimalformed fetus presenting: a) The abdominal mass floats freely in the amniotic fluid. b) The color Doppler indicates a bi-directional blood stream within the exteriorized heart. c) 11 weeks of gestation fetus with amniotic membrane covering the remnant of the exencephalic brain; sever face dismorphism; d) scoliosis; e) large anterior thoraco-abdominal defect, with ectopia cordis and eviscerated lungs, intestines and liver; sever limb malformations.

GmbH \& OHG, Zipf, Austria) using the IC5-9-D probe in the day of admission and repeated at 11weeks 1 day. A single live fetus with a crown-rump length of 25.9 $\mathrm{mm}$ corresponding to 9 weeks 3 days $(<3$ rd percentile) was visualized. Important defects of the thoracic and abdominal walls with protrusion of the heart and abdominal organs were noted (fig 1a). The cerebral tissue and flat bones of the calvaria were absent. The vertebral column was clearly seen and presented a lateral angulation. No sonographic evidence of omphalocele was visible as the abdominal mass floated freely in the amniotic fluid. The amniotic membrane seemed to be adherent to the base of the skull. A relatively small amniotic cavity with a large extra-embryonic coelomic cavity was noted. Amniotic bands were not seen. Additionally, no patent images of the limbs were noted, but dismorphic limb buds with erratic movements were seen. The color Doppler clearly indicated a bi-directional blood stream within the exteriorized heart (fig 1b.). Prenatal genetic investigation was performed at 11 weeks by analysis of circulating free DNA (cfDNA) in maternal blood (Harmony Prenatal Test ${ }^{\circledR}$, Ariosa Diagnostics, Inc. San Jose, CA). The results showed a female fetus with low probability for trisomy 13,18 or 21 , low probability for sexual chromosome aneuploidy and no evidence of 22q11.2 deletion. After informing the patient upon the reserved fetal prognostic and obtaining her written consent the therapeutic abortion was performed using mifepristone and misoprostol. After expulsion, the heart continued to beat for a minute outside the fetus' body (Movie 1, on the jour- nal site). The pathologic exam revealed a $30 \mathrm{~g}$ fetus and confirmed all sonographic findings. The remnant of the exencephalic brain was covered by the amniotic membrane (fig 1c) which was adherent to the placenta. The umbilical cord was short and embedded in an amniotic sheet which connected the skin margin of the skull defect to the placenta. The vertebral column was scoliotic to the left with no spinal dysraphism (fig 1d) and the limb presented multiple anomalies (fig 1e).

\section{Discussion}

The etiology of LBWC is unknown, though some hypotheses were advocated such as, Tropin's amniotic band theory [5] and Van Allen theory of vascular disruption [2]. In our case, the most suitable theory that can explain the unusual combination of anomalies is by the embryonal folding theory of Hartwing [6].

Some authors proposed a genetic ethology involving laterality, but there is little evidence pointing in that direction considering that sibling recurrence is insignificant [7]. In fetuses with body LBWC, the karyotype is usually normal, although a case of maternal uniparental disomy of chromosome 16 and body stalk anomaly has been described [8].

The prenatal diagnosis is made using ultrasonography and the suspicion can be raised in the first trimester [9]. The sonographic hallmarks in the late first trimester and early second trimester are thoraco- and/or abdominoschisis, neural tube abnormalities, severe scoliosis, limb ab- 
normalities, positional deformities, abnormalities of fetal membranes and short or absent umbilical cord [10]. The most characteristic finding of this syndrome is considered to be the scoliosis that can be revealed starting with the first trimester [10]. In cases where fetal position or maternal obesity limits the possibility of a proper ultrasound diagnostic, fetal MRI can facilitate a suitable view of the fetal brain [11], spine, neck, thorax, abdomen and urinary tract and can provided convincing evidence for the early diagnosis of this rare pathology [12].

While early diagnosis of LBWC was described by ultrasound, their findings were not confirmed by histopathological examination [9]. Foremost, the differential diagnosis for our case was pentalogy of Cantrell. While the embryo did align with the description of the pentalogy of Cantrell [13], the additional limb defects, exencephalic brain adherent to the amniotic membrane and large extraembryonic coelomic cavity point in the direction of LBWC.

General prognosis of LBWS is very poor. After accurate ultrasound diagnosis is established, the pregnant woman should be thoroughly informed and counseled about the reserved prognosis of the fetus and lack of recurrence risk, followed by consented therapeutic abortion.

In conclusion, practitioners must be aware of this pathology and the diagnosis can be made at an early gestational age using prenatal sonography. Correct prenatal evaluation even in the first trimester is very important for the parental decision making process.

\section{Bibliography}

1. Torres US, Portela-Oliveira E, Braga Fdel C, Werner H Jr, Daltro PA, Souza AS. When Closure Fails: What the Radiologist Needs to Know About the Embryology, Anatomy, and Prenatal Imaging of Ventral Body Wall Defects. Semin Ultrasound CT MR 2015;36:522-536.
2. Van Allen MI, Curry C, Walden CE, Gallagher L, Patten RM. Limb-body wall complex: II. Limb and spine defects. Am J Med Genet 1987;28:549-565.

3. Russo R, D'Armiento M, Angrisani P, Vecchione R. Limb body wall complex: a critical review and a nosological proposal. Am J Med Genet 1993;47:893-900.

4. Sahinoglu Z, Uludogan M, Arik H, et al. Prenatal ultrasonographical features of limb body wall complex: a review of etiopathogenesis and a new classification. Fetal Pediatr Pathol 2007;26:135-151.

5. Torpin R. Amniochorionic mesoblastic fibrous strings and amnionic bands: associated constricting fetal malformations or fetal death. Am J Obstet Gynecol 1965;91:6575.

6. Hartwig NG, Vermeij-Keers C, De Vries HE, Kagie M, Kragt H. Limb body wall malformation complex: an embryologic etiology? Hum Pathol 1989;20:1071-1077.

7. Gajzer DC, Hirzel AC, Saigal G, Rojas CP, Rodriguez MM. Possible Genetic Origin of Limb-Body Wall Complex. Fetal Pediatr Pathol 2015;34:257-270.

8. Chan Y, Silverman N, Jackson L, Wapner R, Wallerstein R. Maternal uniparental disomy of chromosome 16 and body stalk anomaly. Am J Med Genet 2000;94:284-286.

9. Paul C, Zosmer N, Jurkovic D, Nicolaides K. A case of body stalk anomaly at 10 weeks of gestation. Ultrasound Obstet Gynecol 2001;17:157-159.

10. Becker R, Runkel S, Entezami M. Prenatal diagnosis of body stalk anomaly at 9 weeks of gestation. Case report. Fetal Diagn Ther 2000;15:301-303.

11. Muresan D, Popa R, Stamatian F, Rotar IC. The use of modern ultrasound tridimensional techniques for the evaluation of fetal cerebral midline structures- a practical approach. Med Ultrason 2015;17:235-240.

12. Higuchi T, Sato H, Iida M, Kim SH, Narimatsu Y, Tanaka M. Early second-trimester diagnosis of body stalk anomaly by fetal magnetic resonance imaging. Jpn J Radiol 2013;31:289-292.

13. Grigore M, Iliev G, Gafiteanu D, Cojocaru C. The fetal abdominal wall defects using 2D and 3D ultrasound. Pictorial essay. Med Ultrason 2012;14:341-347. 